\title{
Hierarchical non-linear mixed-effects models for estimating growth parameters of western Mediterranean solitary coral populations
}

\author{
Barbara Cafarelli ${ }^{\mathrm{a}, *}$, Crescenza Calculli $^{\mathrm{a}}$, Daniela Cocchi $^{\mathrm{b}}$, Elettra Pignotti $^{\mathrm{b}}$ \\ ${ }^{a}$ Department of Economics, University of Foggia, largo Papa Giovanni Paolo II 1, Foggia, \\ Italy \\ ${ }^{b}$ Department of Statistical Sciences "Paolo Fortunati", University of Bologna, via Belle \\ Arti 41, Bologna, Italy
}

\begin{abstract}
Marine biologists usually assess coral growth through the von Bertalanffy growth function (VBGF), a function of several biological parameters linked to age by a non-linear relationship. Coral growth parameters are then evaluated via ordinary least squares after a linear transformation of the VBGF. Current literature focuses on linearization techniques, but these methods are often used without considering a careful data examination and the presence of variability in coral of the same age or in coral of the same colony. For these reasons, a more thorough approach based on a hierarchical non-linear mixed-effects model is proposed. This model takes into account the influence of sites characteristics to model heterogeneity between sites. Moreover, the contribution of environmental factors and all the reliable information that may influence coral growth can be suitably modelled. Two model specifications based on the standard and new VBGF parameterizations are introduced to analyse the growth of a solitary coral species Balanophyllia europaea. Results from the proposed modelling approach show the importance of including environmental conditions for species coral growth and support; furthermore, the results show the importance of the possibility of
\end{abstract}

\footnotetext{
* Corresponding author

Email address: barbara.cafarelli@unifg.it (Barbara Cafarelli)
}

Preprint submitted to Ecological Modelling

December 14, 2016 
accounting for variability from different sources in terms of estimated growth curves.

Keywords: solitary corals, von Bertalanffy growth function, parameter estimation, within-between site variability, environmental covariates, uncertainty modelling

2010 MSC: 00-01, 99-00

1

2

\section{Introduction}

In marine biology, demographic parameters of living populations are crucial indicators for investigating the relationships between organisms and their environment and to assess the stability of habitats. In fact, the exploitation of marine resources in fragile ecosystems (coral reefs, coastal bays, and flats of barrier islands) poses some crucial issues for conservation strategies and management purposes. Consequently, marine biologists are very interested in evaluating and monitoring coral growth (Stolarski et al., 2007, Goffredo and Chadwick-Furman, 2000) since the population dynamics of these invertebrates may be considered as an indicator of ecological change and anthropogenic pressure (Ferrigno et al. 2016 Lirman et al., 2014). Individual demographic variables, such as age, oral disk length, and body size, are the basis for modelling the peculiarities of these organisms, as well as the growth and relationships between them and their environment (Ault et al., 2014). It is also relevant to analyse the relationship between coral age and size, as these characteristics are strictly related to reproductive activity that is dependent on how fast corals reach the minimum size to let the planulae exit the oral disk, enabling corals to reproduce. All these considerations highlight the importance of coral growth modelling for the evaluation of habitat stability and provide information on population turnover in order to identify and propose techniques for the restoration of damaged or degraded coastal areas. A popular model used by marine biologists for analysing 
the growth of several marine organisms (Ricker, 1979, Cailliet et al. 2006, LloydJones et al., 2014; Purcell et al. 2016) is the von Bertalanffy growth function (VBGF) curve (von Bertalanffy, 1938). This non-linear growth function links

the size of fish and invertebrates to their age. Recently, the VBGF has been applied to modelling solitary coral growth in the Mediterranean sea (Goffredo and Lasker, 2008; Goffredo et al., 2010, Caroselli et al., 2012, Cafarelli et al., 2016). There are several methods (hereinafter referred to as traditional methods) for estimating the VBGF parameters (Gulland and Holt, 1959, Fabens, 1965, Basso and Kehr, 1991), however, they are not as accurate as desired (McClanahan et al. 2009) and do not exploit statistical reasoning. The common purpose of the traditional methods is a linear transformation of the VBGF in order to pbtain the parameter estimations by ordinary least squares (OLS) (Yee and Barron, 2010). These methods are often applied without considering properties coming from statistical estimation theory (Vonesh and Chinchilli, 1997), the statistical distribution of observed data, or the sampling design. In particular, correlation and variability among corals collected at the same site (within-sites) or in different sites (between-sites) is neglected, thus, inducing errors in parameter estimates. Moreover, environmental site specific characteristics related to genetic and environmental factors, such as sea water temperature (Galli et al. 2016), ultraviolet-B radiation, surface ocean acidification, and human anthropogenic stress (Caroselli et al., 2012), cannot be directly inserted in the VGBF. In order to overcome these limitations, we suggest hierarchical non-linear mixed effects models (HNLMMs) as a more feasible approach to estimate parameters of the von Bertalanffy coral growth function and propose an alternative VBGF parameterization that considers the influence of environmental conditions on the site where individual coral data are collected. Rather than following the growth process of marked individuals over time, we refer to different individu- 
49

50

70 rewriting (1) as

$$
\frac{d L}{d t}=k\left(L_{\infty}-L\right),
$$

where $k$ is the growth rate, which is related to maintenance costs, and $L$ is the length at time $t$. Goffredo et al. (2010) suggested representing corals growth by

$$
y(t)=L_{\infty}\left(1-e^{-K t}\right),
$$


where $y(t)$ is the individual length at age $t, L_{\infty}$ is the asymptotic length representing the maximum theoretical value that a species will tend towards, and $K$ is the constant known as the Brody growth coefficient, i.e., the rate at which growth approaches this asymptote.

Model (2) does not account for the influence of environmental covariates. To this end, in the spirit of Galluci and Quinn (1979), we propose a new parameterization of the VBGF as follows. According to Koojiman (2000), for organisms of the same species with different food availabilities, the logarithm of the VBGF growth rate, $K$, decreases linearly with the asymptotic length, $\ln (k) \propto 1 / L_{\infty}$; thus, different combinations of $K$ and $L_{\infty}$ can give approximately the same fit (as well as high values of $K$ combined with low values of $L_{\infty}$ and vice versa). In particular, Kooijman et al. (2008) pointed out that $L_{\infty}$ can be considered as independent from the environmental conditions, which allows us to consider the following parameterization of $(2)$ :

$$
y(t)=L_{\infty}\left(1-e^{-t e^{\frac{c}{L_{\infty}}}}\right)
$$

where $c=\ln (K) L_{\infty}$. Following a biological perspective, the new parameter $c$ introduced in (3) can be seen as the part of individual length growth linked to site-specific conditions such as environmental factors. Compared to the traditional specification of the VBGF in (2), (3) accounts for the global effects of site-specific environmental covariates by means of the new parameter $c$; consequently, it also obtains a more reliable result in terms of model estimation.

The validity of the proposal of curves such as $(2)$ and $(3)$ is essentially descriptive. Moreover, the curve can be proposed for the growth of an individual, but also holds for aggregated cases if the $y$ values have the meaning of group averages. 


\section{Different approaches for estimating VBGF parameters}

The parameter estimation characterizing the growth of coral populations can be achieved by means of traditional methods or the HNLMMs proposed herein.

\subsection{Traditional methods}

The methods broadly used for estimating the VBGF parameters are the Gulland-and-Holt (GH) plot (Gulland and Holt, 1959), size-increment method proposed by Fabens (1965), linearization proposed by Basso and Kehr (1991), and the parameterization by Galluci and Quinn (1979). Each method proposes a re-parameterization of 2 in order to obtain linear regression models that use the OLS method to estimate parameters. These methods are easy to implement but imply several limiting hypotheses. First, traditional methods do not take into account the grouped-structure of data collected in situ, and consequently, the association usually expressed by the linear correlation among corals sampled at the same site. In this way, the Gauss-Markov uncorrelated residuals hypothesis, required for linear regression models, is violated. Moreover, ignoring data grouped-structure leads to an overall VBGF parameter estimation, common to the entire population, without the possibility for obtaining site-specific estimates. For any association between $L_{\infty}$ and $K$, another limitation is not explicitly considering the influence of environmental parameters such as the sea surface temperature, sea current, solar radiation, and the variability at the coral, colony, and site levels. The above limits and the forced linearization required for using traditional methods may lead to a bias in the VBGF parameter estimates.

\subsection{HNLMM approach}

In order to overcome the previous drawbacks, HNLMMs are a suitable solution. These models are used in a wide range of subject-matter studies, e.g., biological, agricultural, environmental, and medical applications (Paul and Saha, 
2007), especially since suitable software is now available. In particular, they are a natural way to analyse grouped, repeated measures, multilevel data, and block designs.

The HNLMM approach may be regarded as a model formulation that can handle data from several individuals linked to common conditions and suitably consider a non-linear response function (Burnett et al., 1995, Cressie et al. 2009). As in the hierarchical linear case, within- and between-individual variations are accommodated within the framework of a two-stage model (Lindstrom and Bates, 1990). More precisely, at the first stage, which models individual (coral) data, the within-site behavior is characterized by a non-linear regression model based on the VBGF, and the within-site covariance structure is specified by modelling the error term distribution. The between-site variability is represented in the second stage through site-specific regression parameters, which also may incorporate both systematic and random effects (Davidian and Giltinan, 1995). Thus, following Lindstrom and Bates (1990), at the first stage of the HNLMM for the solitary coral growth curve based on the VBGF, the length of the $j$-th coral on the $i$-th site is modelled by

$$
y_{i j}=f\left(\phi_{i}, \boldsymbol{x}_{i j}\right)+\varepsilon_{i j} \quad i=1, \ldots, M \quad j=1, \ldots, n_{i},
$$

where $n_{i}$ is the number of corals in each of $M$ sites, $f$ is the VBGF, $\phi_{i}$ is the site-specific parameter vector, $\boldsymbol{x}_{i j}$ is the individual covariates vector, and $\varepsilon_{i j}$ is the individual random error. Hereinafter, for simplicity, we refer to a set of two covariates that may affect coral dimensions at a site; specifically, we consider the annual mean sea surface temperature gradient, $T$, and the mean solar radiation gradient, $R$, so each $\boldsymbol{x}_{i j}$ individual vector is bi-dimensional.

Following Pinheiro and Bates (2000), by posing $\varepsilon_{i} \sim N\left(\mathbf{0}, \sigma_{\varepsilon}^{2} \boldsymbol{\Lambda}_{i}\right)$, where $\boldsymbol{\Lambda}_{i}$ is a positive-defined matrix whose dimension depends on the number of 
observations in each site, it is possible to allow heteroscedastic and correlated within-site errors. Moreover, the decomposition of $\boldsymbol{\Lambda}_{i}$ into a variance structure component and correlation structure component allows us to model the two structures separately and combine them into a flexible family of models for the within-site variance-covariance. The normality assumption for the errors is motivated by physical and biochemical considerations on coral growth and by the fact that the data consists of repeated measurements Lindstrom and Bates, 1990). Moreover, the inclusion of $\boldsymbol{\Lambda}_{i}$ into the model allows for the specification of a non-independent marginal correlation structure, i.e., the $\operatorname{AR}(1)$ correlation (Box et al. 2008). In fact, for the data under consideration, the assumption that errors have a common variance, $\boldsymbol{\Lambda}_{i}=\mathbf{I}_{i}$, is unrealistic mainly for two reasons. First, young corals are less variable than older corals because environmental factors have less time to influence them. The second reason depends on how age measurements are taken. Since the adopted non-invasive way of determining the age of corals counts growth rings, measurements may be less precise in the youngest and smallest corals than in the oldest. For young corals, counting the ultimate rings is difficult because their thickness is quite small and identification is not always precise.

At the second stage, the site-specific parameter vector is modelled by

$$
\boldsymbol{\phi}_{i}=\mathbf{A}_{i} \boldsymbol{\beta}+\mathbf{b}_{i} \quad \mathbf{b}_{i} \sim N\left(\mathbf{0}, \sigma_{b}^{2} \mathbf{D}\right)
$$

where $\mathbf{A}_{i}$ is the design matrix of fixed effects, $\boldsymbol{\beta}$ is a $p$-dimensional vector of fixed effects, $\mathbf{b}_{i}$ is a random effects vector associated with the $i$-th site whose dimension depends on the number of $\phi$ components, and $\sigma_{b}^{2} \mathbf{D}$ is a general variance-covariance matrix. It is also assumed that the observations coming from different sites are mutually independent and the error term, $\varepsilon_{i}$, and is independent of the random effect, $\mathbf{b}_{i}$ (Gelman and Hill, 2007). 


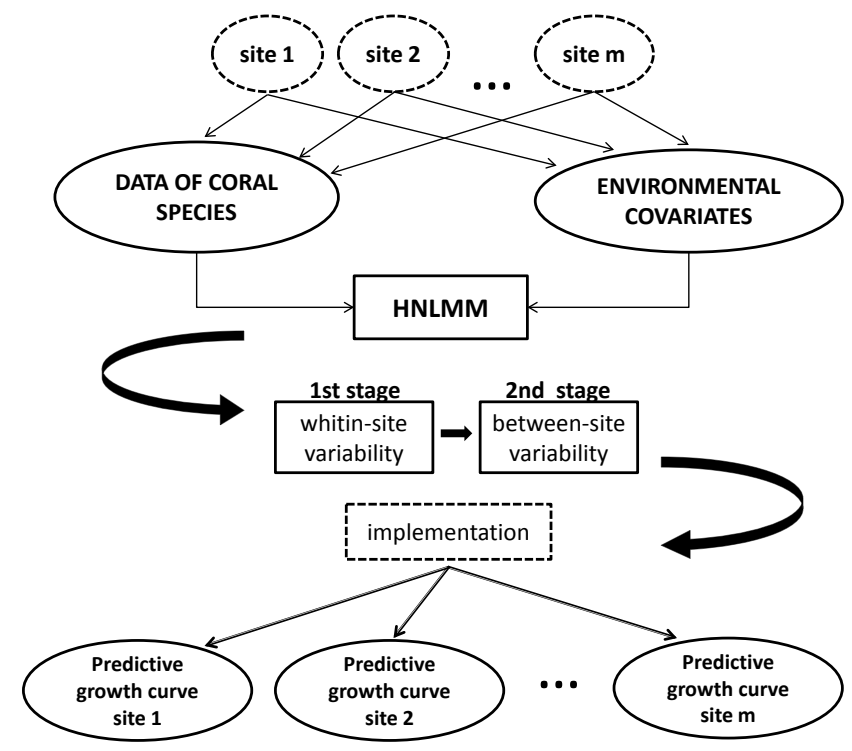

Figure 1: Conceptual diagram of the HNLMM approach for coral growth estimation.

The HNLMM parameters are estimated by means of the nlme function of $R$ software (Pinheiro et al. 2016) for the model implementation under the two different parameterizations. A synthetic conceptual diagram that summarizes the modelling approach for the estimation of coral growth is proposed in Figure 1 .

\subsubsection{Standard parameterization}

According to (2) and (4), the solitary coral growth curve for the $j$-th coral on the $i$-th site is modelled at the first stage of the hierarchy by

$$
y_{i j}=L_{\infty_{i}}\left(1-e^{-K_{i} t_{i j}}\right)+\varepsilon_{i j} .
$$


178 For each site, $\phi_{i}=\left[L_{\infty_{i}}, K_{i}\right]^{\prime}$ is a bi-dimensional vector of parameters specified 179 by recalling (5) as follows:

$$
\begin{aligned}
\mathbf{A}_{i} & =\left(\begin{array}{cccccc}
1 & R_{i} & T_{i} & 0 & 0 & 0 \\
0 & 0 & 0 & 1 & R_{i} & T_{i}
\end{array}\right), \\
\boldsymbol{\beta} & =\left(\begin{array}{llllll}
L_{\infty} & a_{1} & a_{2} & K & a_{3} & a_{4}
\end{array}\right)^{\prime}, \\
\mathbf{b}_{i} & =\left(\begin{array}{ll}
b_{1 i} & b_{2 i}
\end{array}\right)^{\prime},
\end{aligned}
$$

with $\boldsymbol{\phi}_{i} \sim N\left(\mathbf{A}_{i} \boldsymbol{\beta}, \sigma_{b}^{2} \mathbf{D}\right)$. Consequently, the components of $\boldsymbol{\phi}_{i}$ can be expressed by

$$
\begin{gathered}
L_{\infty i}=L_{\infty}+a_{1} R_{i}+a_{2} T_{i}+b_{1 i}, \\
K_{i}=K+a_{3} R_{i}+a_{4} T_{i}+b_{2 i} .
\end{gathered}
$$

\subsubsection{New parameterization}

Following (3) and (4), the formulation of the non-linear mixed effects model of the VBGF for solitary corals is

$$
y_{i j}=L_{\infty_{i}}\left(1-e^{-t_{i j} e^{\frac{c_{i}}{L_{\infty i}}}}\right)+\varepsilon_{i j} .
$$

3ere, the bi-dimensional parameter vector is $\phi_{i}=\left[L_{\infty_{i}}, c_{i}\right]^{\prime}$. Unlike (6), only parameter $c_{i}$ is affected by the environmental factors, whereas $L_{\infty i}$ is characterized by genetic and not site-dependent features as suggested in Kooijman et al. (2008). This corresponds to slightly different definitions of the quantities in (7) 
and $(8)$ as follows:

$$
\begin{aligned}
\mathbf{A}_{i} & =\left(\begin{array}{cccc}
1 & 0 & 0 & 0 \\
0 & 1 & R_{i} & T_{i}
\end{array}\right), \\
\boldsymbol{\beta} & =\left(\begin{array}{llll}
L_{\infty} & c & a_{3} & a_{4}
\end{array}\right)^{\prime}, \\
\mathbf{b}_{i} & =\left(\begin{array}{ll}
b_{1 i} & b_{2 i}
\end{array}\right)^{\prime} .
\end{aligned}
$$

Thus, the parameter vector is now $\phi_{i} \sim \mathrm{N}\left(\mathbf{A}_{i} \boldsymbol{\beta}, \sigma_{b}^{2} \mathbf{D}\right)$ with

$$
\begin{aligned}
L_{\infty i} & =L_{\infty}+b_{1 i}, \\
c_{i} & =c+a_{3} R_{i}+a_{4} T_{i}+b_{2 i} .
\end{aligned}
$$

In this way, the influence of covariates is correctly ascribed only to parameter c. This parameterization, compared to the traditional one, has the advantage of isolating the parameter sensible to environmental influences so that it is possible to obtain a more meaningful and parsimonious statistical model when covariates are involved. The deterministic methods used by biologists are not suitable for this parameterization because they were designed to find $K$ and $L_{\infty}$, while the new parameterization does not contain $K$.

\section{Analysing Mediterranean solitary coral data}

\subsection{Data}

In this study, a species of solitary scleractinian coral is analysed, Balanophyllia europaea, that lives on a rocky substratum at a depth range of $0-50 \mathrm{~m}$. Its wide distribution in the Mediterranean basin and demographic characteristics closely related to the environmental conditions allow for an assessment of habitat stability and suitability with regards to climatic changes and human anthropogenic stress (Caroselli et al., 2012). The dataset used comes from Goffredo 


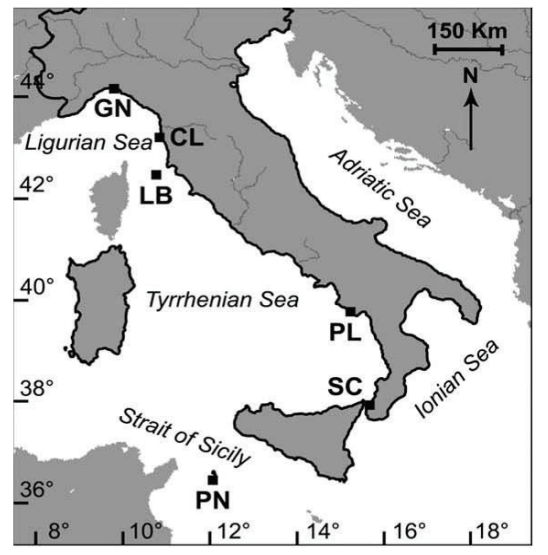

Figure 2: Site locations: Genova (GN), Calafuria (CL), Elba Isle (LB), Palinuro (PL), Scilla (SC), Pantelleria Isle (PN). (Caroselli et al., 2012)

et al. (2007) and Goffredo and Lasker (2008). From $9^{\text {th }}$ November 2003 to $30^{\text {th }}$ September 2005, samples of the coral species were collected separately at different times from six Italian sites, specifically, Genova, Calafuria, Elba, Palinuro, Scilla, and Pantelleria, as illustrated in Figure 2 the samples were taken from Caroselli et al. (2012) at a maximum biomass density depth of 15-17 m along a latitudinal gradient of $44^{\circ} 20^{\prime} \mathrm{N}-36^{\circ} 45^{\prime} \mathrm{N}$. A detailed description of protocol procedures and measurements can be found in Caroselli et al. (2012).

The measurements considered for this case study are:

- The corallite length in $m m$ ( $L$, maximum axis of the oral disc) measured by a calliper.

- The ages, in years, of corals computed as the mean over three repeated counts of the growth bands of the skeleton via computerized tomography (CT) scans.

For each site, two environmental covariates are considered related to data availability: 


\begin{tabular}{|c|c|c|c|c|c|c|}
\hline & Genova (C) & Calafuria (C) & Elba (I) & Palinuro $(\mathrm{C})$ & Scilla (C) & Pantelleria (I) \\
\hline$n$ of sampled individuals & 42 & 34 & 34 & 54 & 32 & 42 \\
\hline $\begin{array}{l}\text { mean age (years) } \\
(95 \% \mathrm{CI})\end{array}$ & $\begin{array}{c}7.4 \\
(6.4-8.5)\end{array}$ & $\left(4.9^{5.5}-6.2\right)$ & $\begin{array}{c}4.6 \\
(3.9-5.4)\end{array}$ & $\begin{array}{c}6.9 \\
(6.0-7.8)\end{array}$ & $\begin{array}{c}6.2 \\
(5.3-7.2)\end{array}$ & $\begin{array}{c}5.2 \\
(4.5-6.0)\end{array}$ \\
\hline $\begin{array}{l}\text { mean length }(\mathrm{mm}) \\
(95 \% \mathrm{CI})\end{array}$ & $\begin{array}{c}11.7 \\
(10.4-12.9)\end{array}$ & $\begin{array}{c}8.3 \\
(7.4-9.3)\end{array}$ & $\begin{array}{c}9.0 \\
(7.9-10.0)\end{array}$ & $\begin{array}{c}9.9 \\
(9.0-10.8)\end{array}$ & $\begin{array}{c}9.9 \\
(8.8-10.9)\end{array}$ & $\begin{array}{c}8.8 \\
(8.0-9.7)\end{array}$ \\
\hline $\mathrm{R}\left(W / m^{2}\right)$, annual mean $(S E)$ & $166.95(1.02)$ & $170.07(1.02)$ & $172.74(1.02)$ & $181.48(1.01)$ & $187.31(1.02)$ & $192.95(1.02)$ \\
\hline $\mathrm{T}\left({ }^{\circ} \mathrm{C}\right)$, annual mean $(S E)$ & $19.56(0.04)$ & $18.02(0.04)$ & $18.74(0.04)$ & $19.14(0.03)$ & $19.54(0.02)$ & $19.88(0.04)$ \\
\hline
\end{tabular}

Table 1: Descriptive statistics of Balanophyllia europaea samples and annual averages of environmental indicators per site. R: Solar Radiation (from $190 \mathrm{~W} / \mathrm{m}^{2}$ ); T: Sea Surface Temperature (from $18^{\circ} \mathrm{C}$ ). Site typology: coast (C) and isle (I).

- The annual mean sea surface temperature gradient $T$ obtained from the National Mareographic Network of the Agency for the National System for Environmental Protection (ISPRA), available at http://www . isprambiente . gov.it/.

- The mean solar radiation gradient $R$ obtained from the International Cloud Climatology Project (ISCCP), available at http://www.ingrid. ldgo.columbia.edu/

In Table 1 , some basic descriptive statistics and the annual averages of main environmental indicators are reported for each site. The coral length distributions at various ages with different growth rate patterns for the six sites are shown in Figure 3 .

\subsection{Statistical analysis}

Traditional methods, i.e., the GH plot (Gulland and Holt, 1959), size-increment method (Fabens, 1965), and proposal by Basso and Kehr (1991), are first used to estimate the VBGF parameters for solitary coral data. The goodness of fit is tested by graphical inspection of standardized residuals at the population level and by coefficients of determination, $R^{2}$.

An HNLMM is estimated for each of the two parameterizations in Section 3.2 In order to identify the optimal models that balance between model 

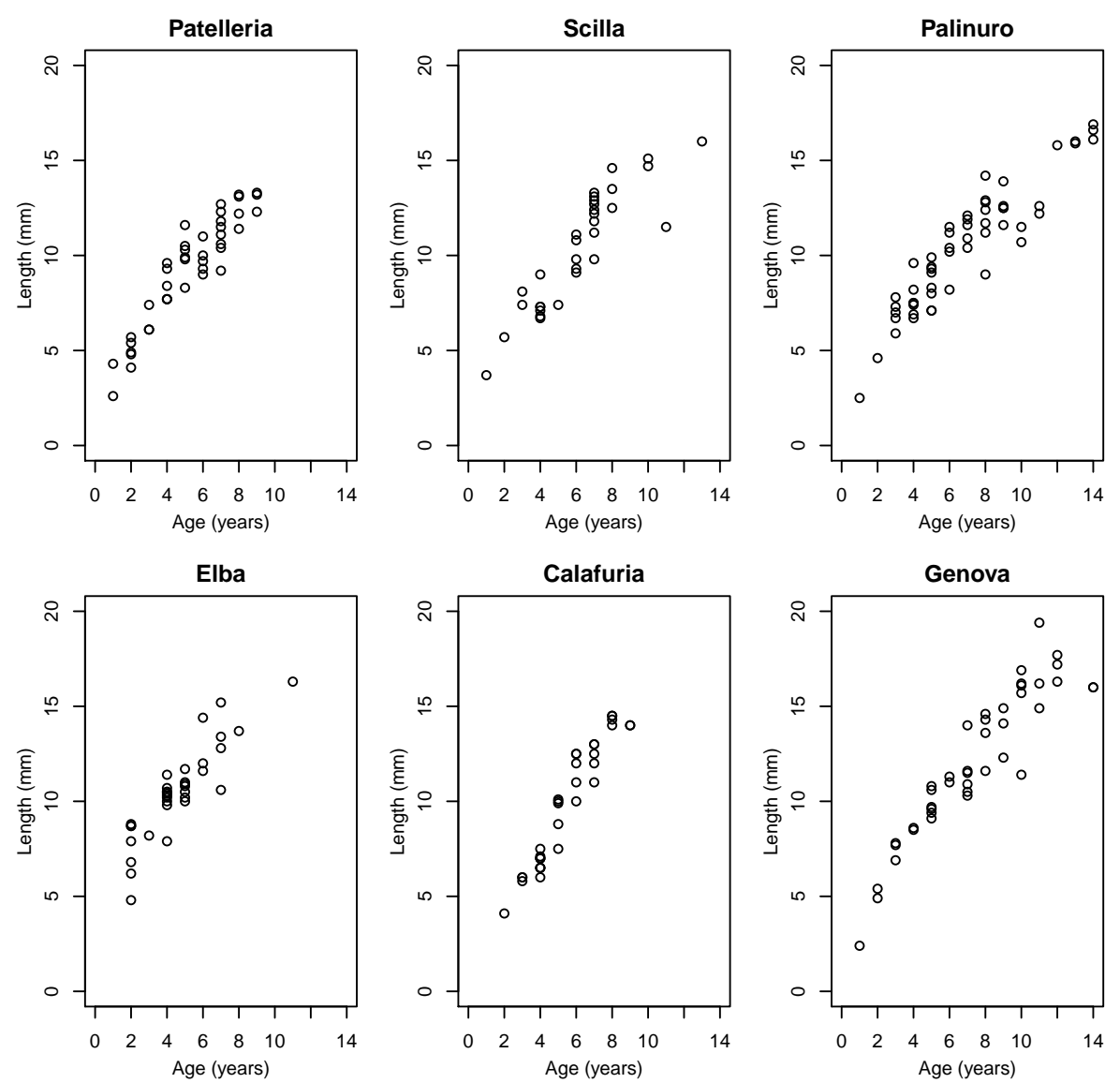

Figure 3: Site scatterplots of length versus age.

fit and complexity, candidate models are compared using the Bayesian information criterion (BIC) (Schwarz, 1978) and the Akaike information criterion (AIC) (Sakamoto et al., 1986). For the two alternative approaches, the results of this comparison, available in Pignotti (2013), lead to the best model specifications; $\left(L_{\infty i}, K_{i}+b_{2 i}\right)^{\prime}$ for the standard parameterization and $\left(L_{\infty i}+b_{1 i}, c_{i}\right)^{\prime}$ for the new parameterization. The variance among corals at the same site is modelled for the standard specification as a combination of an exponential increasing function and a negative power function, whereas that of the new parameterization is modelled as a mixture of a constant function and a positive power function. 
These choices allow us to neglect the influence of the extreme fitted values linked to the young and old coral measurements, about which difficulties in counting the growth rings usually arise. For both parameterizations, an autoregressive $\mathrm{AR}(1)$ correlation structure is adopted.

The results of the best models, coming from traditional methods and the HNLMM approach, are compared using three cross-validation techniques, $\mathrm{CV}_{1}$, $\mathrm{CV}_{2}$, and $\mathrm{CV}_{3}$ suggested by Carroll and Cressie (1996).

In particular, $\mathrm{CV}_{1}$ is used to assess the unbiasedness of the predictor (optimal value: $\left.\mathrm{CV}_{1}=0\right), \mathrm{CV}_{2}$ is used to assess the accuracy of the mean squared prediction error (optimal value: $\mathrm{CV}_{2}=1$ ), and $\mathrm{CV}_{3}$ is used to check the goodness of the prediction (small values of $\mathrm{CV}_{3}$ indicate a good fit).

\subsection{Results from traditional methods}

The estimates of VBGF growth parameters, $\hat{L}_{\infty}$ and $\hat{K}$, obtained from the traditional linearization methods with parameterization (2) are reported in Table 2. Estimates are obtained by considering corals from unique sites without differentiating the six different sites along the latitude gradient. As expected, the traditional methods (Gulland and Holt, 1959, Fabens, 1965, Basso and Kehr, 1991) underestimate both young (age $<4$ years) and old (age $>11$ years) corals (Figure 4. left panels). Furthermore, the residual examination reported in Figure 4 (right panels) highlights non-random patterns in data distribution, which suggests looking for a better fit via non-linear models. Furthermore, we consider the variability at the individual, colony, and site levels and the explicit influence of specific environmental components. Values of $R^{2}$ suggest that the size-increment method proposed by Fabens (1965) is the best model in terms of goodness of fit (Table 2. 
Gulland and Holt
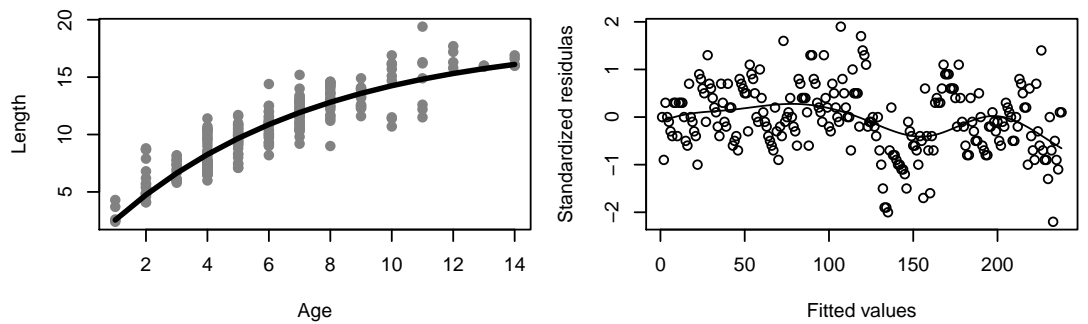

Fabens
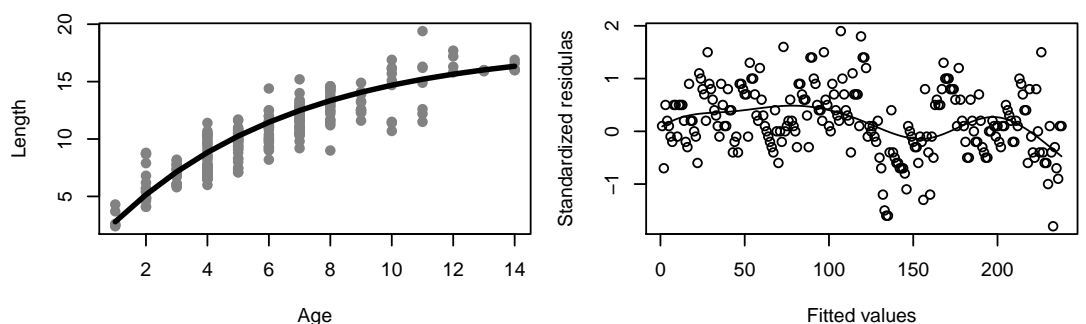

Basso and Kehr
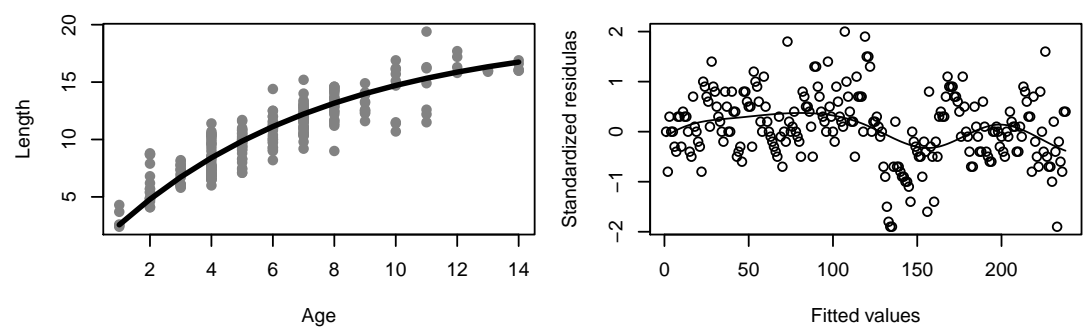

Figure 4: Growth curves (left panels) and residuals (right panels) using traditional estimation methods.

\subsection{Results from HNLMMs}

According to the alternative HNLMM specifications in (8) and (11), fixed and random effects components for growth data are estimated and reported in Tables 34

For the standard parameterization in Table 3 regression coefficients $\hat{a}_{1}$ and $\hat{a}_{2}$ of (8) related to $R$ and $T$ covariates, respectively, suggest that the ultimate length $\hat{L}_{\infty i}$ decreases linearly with both the solar radiation and sea surface temperature. Conversely, estimated coefficients $\hat{a}_{3}$ and $\hat{a}_{4}$ are very close to zero and do not influence the growth rate $K_{i}$. For the new parameterization, the 


\begin{tabular}{lccc}
\hline Linearization Method & $\hat{L}_{\infty}$ & $\hat{K}$ & $R^{2}$ \\
\hline Gulland and Holt (1959) & 18.38 & 0.15 & 0.26 \\
Fabens (1965) & 18.06 & 0.17 & 0.93 \\
Basso and Kehr (1991) & 19.40 & 0.14 & 0.58 \\
\hline
\end{tabular}

Table 2: Estimated VBGF parameters under linearization and coefficients of determination.

\begin{tabular}{|c|c|c|c|c|c|}
\hline & & \multicolumn{2}{|c|}{ HNLMM 8} & \multicolumn{2}{|c|}{ HNLMM 11 } \\
\hline & & Est. & $S E$ & Est. & $S E$ \\
\hline \multirow{7}{*}{ Fixed effects } & $\hat{L}_{\infty}$ & 18.10 & 1.20 & 17.00 & 1.30 \\
\hline & $\hat{a}_{1}$ & -0.08 & 0.09 & & \\
\hline & $\hat{a}_{2}$ & -2.74 & 1.90 & & \\
\hline & $\hat{K}$ & 0.16 & 0.02 & & \\
\hline & $\hat{c}$ & & & -30.10 & 5.00 \\
\hline & $\hat{a}_{3}$ & 0.00 & 0.00 & -0.19 & 0.08 \\
\hline & $\hat{a}_{4}$ & 0.04 & 0.03 & 0.02 & 1.20 \\
\hline
\end{tabular}

Table 3: Fixed effects estimates under the parameterizations 2 -3 .

estimated fixed effects $\hat{a}_{3}$ and $\hat{a}_{4}$ in (11) for the growth coefficient $\hat{c}_{i}$ suggest that growth decreases with the solar radiation $R$ and increases with the sea surface temperature, $T$. In southern colder sites, slower coral growth is more likely to occur than in northern warmer sites as confirmed in Goffredo et al. (2008). The slope of $R$ is higher and slightly significant.

Table 4 shows the estimated random effects for both HNLMMs. As discussed in Section 3.2 introducing random effects allows us to obtain site-specific growth curves. In particular, the estimated random effects are quite large for the new parameterization; this confirms the importance of considering random effect estimates for calibrating growth curves for corals among different sites. Only after considering random effects is it possible to recognize similar behaviour in the two islands Elba and Pantelleria and the stronger-current site Scilla, which suggests future model enrichment including the sea current, for example, as an additional environmental covariate.

Furthermore, Figures 56 show the predicted curves when the within-site 


\begin{tabular}{llrc}
\hline & & HNLMM $(8)$ & HNLMM $\sqrt{11}$ \\
\hline \multirow{6}{*}{ Random effects } & $\hat{b}_{1(G N)}$ & 0.01 & -5.40 \\
& $\hat{b}_{1(C L)}$ & -0.01 & -14.70 \\
& $\hat{b}_{1(L B)}$ & 0.03 & 14.00 \\
& $\hat{b}_{1(P L)}$ & -0.02 & -5.50 \\
& $\hat{b}_{1(S C)}$ & 0.00 & 3.40 \\
& $\hat{b}_{1(P N)}$ & 0.01 & 8.20 \\
& $\hat{b}_{2(G N)}$ & - & 1.30 \\
& $\hat{b}_{2(C L)}$ & - & 3.50 \\
& $\hat{b}_{2(L B)}$ & - & -3.30 \\
& $\hat{b}_{2(P L)}$ & - & 1.30 \\
& $\hat{b}_{2(S C)}$ & - & -0.80 \\
& $\hat{b}_{2(P N)}$ & - & -1.90 \\
\hline \multirow{2}{*}{ Variance } & $\hat{\varphi}_{1}$ & 0.15 & 13864.00 \\
\hline Correlation & $\hat{\varphi}_{2}$ & -0.86 & 3.5 \\
\hline
\end{tabular}

Table 4: Random effects, variance, and covariance estimates under parameterizations 22-3. Here, we use the same site acronyms as in Figure 2.

random effect adjustment is included. Both the population predictions (corresponding to random effects equal to zero) and the within-site predictions (obtained using the estimated random effects from HNLMMs) are displayed in each panel. As shown, accounting for variability coming from differences among sites provides an improvement of fitted curves in some sites (Figure 5) such as Palinuro and Elba. For the new parameterization (Figure 6), remarkable differences in terms of fitted curves can be appreciated in the Elba, Calafuria, and Pantelleria sites even if a worse fitted growth curve occurs for the Elba site. This can be attributed to the lack of environmental information (sea current covariate, for instance), which neutralizes the advantages of the new parameterization. Parameter $c$, conceived as a tool to capture the implicit effect of covariates, is in fact more sensitive to poor environmental information. The standard parameterization, distributing the covariate influences between the two parameters, is 
able to manage this lack of information.

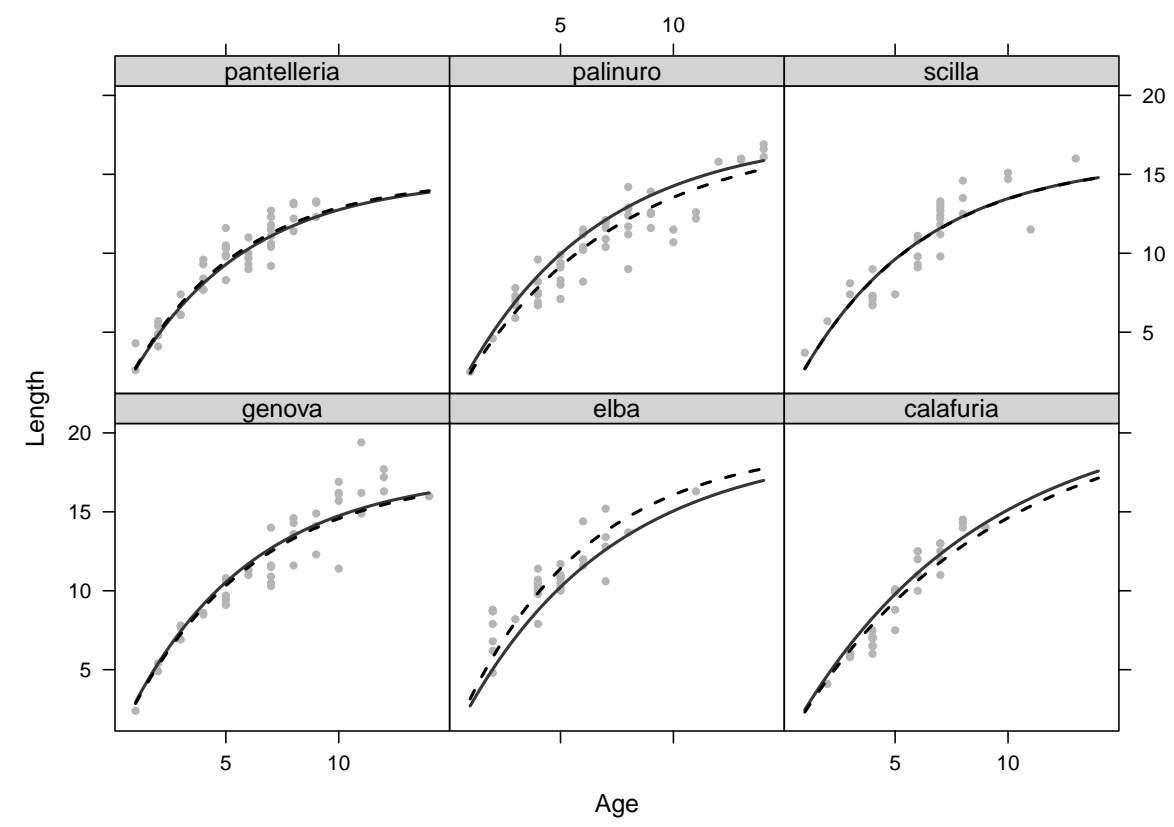

Figure 5: Estimated growth curves under the HNLMM standard parameterization: population prediction level (solid line), site prediction level (dashed line), and observed data (dots).

Model performance under the two parameterizations is compared in Table 5 The new parameterization in (11) has slightly smaller AIC and BIC values suggesting a moderate overall superiority of this parameterization compared to the standard one. Moreover, the graphical inspection of estimated residuals of both models (not reported here) shows random dispersed distributions, confirming the general good performance of the proposed HNLMMs.

\begin{tabular}{lcc}
\hline & AIC & BIC \\
\hline Standard parameterization & 758 & 796 \\
New parametrization & 753 & 791 \\
\hline
\end{tabular}

Table 5: Model comparison for the two parameterizations. 


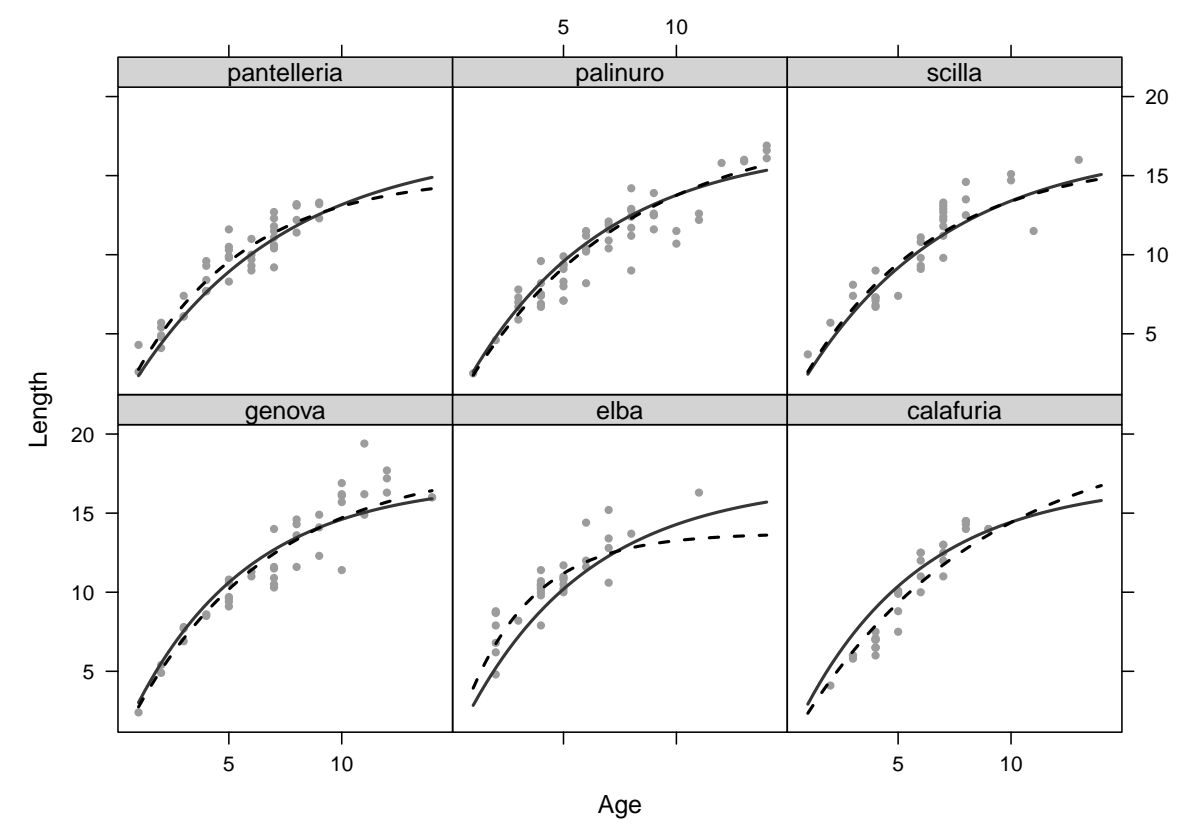

Figure 6: Estimated growth curves under the new HNLMM parameterization: population prediction level (solid line), site prediction level (dashed line), and observed data (dots).

\subsection{Results comparison}

The results comparison confirms that traditional methods lead to a bias in parameter estimates. The estimates from the newly parameterized HNLMM are preferred to those of the increment-size method (Fabens, 1965) with respect to the unbiased nature of the predictor $\left(\mathrm{CV}_{1}\right)$, accuracy of the mean squared prediction $\left(\mathrm{CV}_{2}\right)$, and goodness of fit $\left(\mathrm{CV}_{3}\right)$ for this data (Table 6). These results are also confirmed by graphical inspection in Figure 7. As shown, the prediction at the site level enables us to capture the effect of site-specific features justifying the effort of a complex model versus higher accuracy of estimates. 


\begin{tabular}{l|ccc}
\hline Estimation methods & $\mathrm{CV}_{1}$ & $\mathrm{CV}_{2}$ & $\mathrm{CV}_{3}$ \\
\hline Fabens (1965) & 1.09 & 4.32 & 4.94 \\
HNLMM 111) & 0.05 & 1.17 & 1.40 \\
\hline
\end{tabular}

Table 6: Cross-validation results for the size-increment method and HNLMM 11.

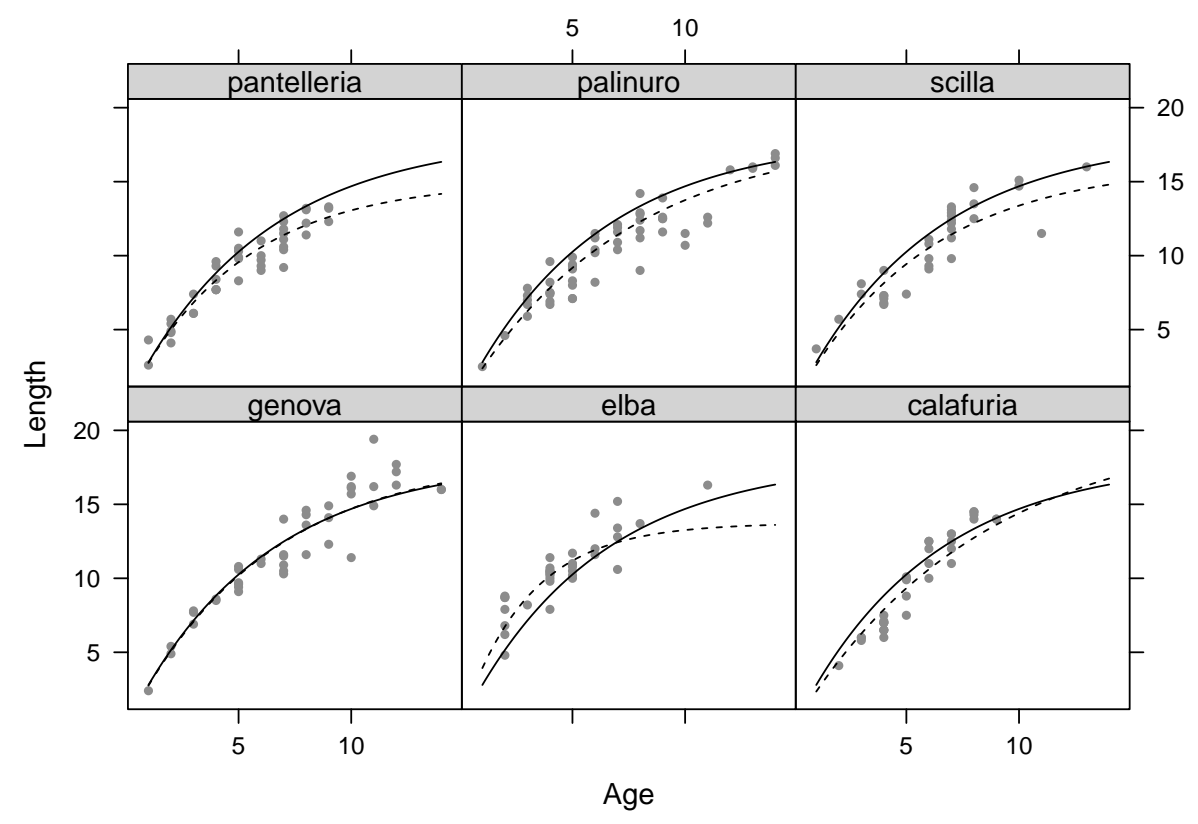

Figure 7: Fitted curves for Fabens' size-increment method (solid line), HNLMM 11] (dashed line), and observed data (dots).

\section{Discussion and conclusions}

In this paper, we introduced a reliable approach for estimating VBGF coral growth parameters, $L_{\infty}$ and $K$, which allows us to overcome the main limitations related to the use of traditional methods. These latter methods are easy to implement but are often proposed ignoring the hierarchical structure that typically characterizes data from marine populations. This leads to inappropriate statistical inference, since they neglect the fact that observations measured within a level (e.g., measurements within the same site) are more 
similar to each other compared to observations obtained between levels (e.g., measurements in different sites). This point is not considered when VBGF parameters are estimated according to traditional methods, which are proposed for assessing information about the entire coral population without addressing differences among sites characterized by environmental conditions and site-specific individual coral features. Conversely, the proposed HNLMM provides several advantages over the more commonly-used OLS approaches when data possess a hierarchical structure. In particular, this approach enables us to consider the influence of select site characteristics, such as locations, typology of site, etc., on overall coral growth and to model within-site measurement correlation and different variabilities at sites. Moreover, it avoids the forced linearization requested by the methods currently employed by biologists, which may lead to a bias in the VBGF parameter estimates; additionally, it simultaneously incorporates environmental information of sites where corals are collected. For the case study concerning the solitary coral species Balanophyllia europaea, the limits of traditional methods are evident. Instead, the two implemented HNLMMs, under the standard and new parameterizations, exhibit clear advantages. In particular, the second parameterization, based on the theory of the energy balance that states the linear correlation of the two growth parameters and the independence of the ultimate length $L_{\infty}$ from the influence of environmental covariates, introduces a new parameter $c$ that describes the growth that can be attributed to site-specific conditions such as environmental factors. The new parameterization leads to a tiny improvement with respect to the first one, which is theoretically more suitable if environmental covariates are introduced in the model. In conclusion, the proposed HNLMM approach, under both parameterizations, suitably fits the hierarchical nature that environmental data collected from different sites possess. In particular, it responds to the need of collectively 
modelling coral distributions from different sites and different site characteristics since mixed-effect modelling permits the use of all available information and manages the variability between individuals. All these considerations make the proposed HNLMMs very attractive tools for growth parameter estimates that overcome the estimates proposed by traditional methods.

\section{Acknowledgements}

This work is developed under the PRIN2015 supported-project "Environmental processes and human activities: capturing their interactions via statistical methods (EPHASTAT)" funded by MIUR (Italian Ministry of Education, University and Scientific Research).

\section{References}

Ault, J.S., Smith, S.G., Browder, J.A., Nuttle, W., Franklin, E.C., Luo, J., DiNardo, G.T., Bohnsack, J.A., 2014. Indicators for assessing the ecological dynamics and sustainability of southern florida's coral reef and coastal fisheries. Ecological Indicators 44, 164-172.

Basso, N.G., Kehr, A.I., 1991. Postmetamorphic growth and population structure of the frog Leptodactylus latinasus (anura: Leptodactylidae). Studies on Neotropical Fauna and Environment 26, 39-44.

Box, G., G.Jenkins, Reinsel, G., 2008. Time Series Analysis: Forecasting and Control. John Wiley, Hoboken, N.J.

Burnett, R.T., Ross, W.H., Krewski, D., 1995. Non-linear mixed regression models. Environmetrics 6, 86-99. 
Cafarelli, B., Calculli, C., Cocchi, D., Pignotti, E., 2016. Modeling multi-site individual corals growth, in: M. Pratesi and C. Pena (Editors). Proceedings of the 48th scientific meeting of the Italian Statistical Society.

Cailliet, G.M., Smith, W.D., Mollet, H.F., Goldman, K.J., 2006. Age and growth studies of chondrichthyan fishes: the need for consistency in terminology, verification, validation, and growth function fitting. Environmental Biology of Fishes 77, 211-228.

Caroselli, E., Zaccanti, F., Mattioli, G., Falini, G., Levy, O., Dubinsky, Z., Goffredo, S., 2012. Growth and demography of the solitary scleractinian coral Leptopsammia pruvoti along a sea surface temperature gradient in the Mediterranean Sea. PLoS ONE 7, e37848. Doi: 10.1371/journal.pone. 0037848 .

Carroll, S., Cressie, N., 1996. A comparison of geostatistical methodologies used to estimate snow water equivalent. Water Resour. Bull. 32, 267278.

Cressie, N., Calder, C.A., Clark, J.S., Hoef, J.M.V., Wikle, C.K., 2009. Accounting for uncertainty in ecological analysis: the strengths and limitations of hierarchical statistical modeling. Ecological Applications 19, 553-570. Doi:10.1890/07-0744.1.

Davidian, M., Giltinan, D., 1995. Nonlinear Models for Repeated Measurement Data. Chapman and Hall, New York, NY.

Fabens, A.J., 1965. Properties and fitting of the von Bertalanffy growth curve. Growth 29, 265-289.

Ferrigno, F., Bianchi, C.N., Lasagna, R., Morri, C., Russo, G.F., Sandulli, R., 2016. Corals in high diversity reefs resist human impact. Ecological Indicators 70, 106-113. 
Galli, G., Bramanti, L., Priori, C., Rossi, S., Santangelo, G., Tsounis, G., Solidoro, C., 2016. Modelling red coral (corallium rubrum) growth in response to temperature and nutrition. Ecological Modelling 337, 137-148.

Galluci, V.F., Quinn, T.J., 1979. Reparameterizing, fitting and testing a simple growth-model. Transactions of the American Fisheries Society 108, 14-25.

Gelman, A., Hill, J., 2007. Data Analysis Using Regression and Multilevel/Hierarchical Models. Cambridge University Press, New York, NY.

Goffredo, S., Caroselli, E., Mattioli, G., Pignotti, E., Zaccanti, F., 2008. Relationships between growth, population structure and sea surface temperature in the temperate solitary coral Balanophyllia europaea (scleractinia, dendrophylliidae). Coral Reefs 27, 623-632.

Goffredo, S., Caroselli, E., Mattioli, G., Zaccanti, F., 2010. Growth and population dynamic model for the non-zooxanthellate temperate solitary coral Leptopsammia pruvoti (Scleractinia, Dendrophylliidae). Marine Biology 157, 2603-2612.

Goffredo, S., Caroselli, E., Pignotti, E., Mattioli, G., Zaccanti., F., 2007. Variation in biometry and demography of solitary corals with environmental factors in the Mediterranean Sea. Marine Biology 152, 351-361.

Goffredo, S., Chadwick-Furman, N., 2000. Abundance and distribution of mushroom corals (scleractinia, fungiidae) on a coral reef at Eilat, northern Red Sea. Bulletin of Marine Science 66, 241-254.

Goffredo, S., Lasker, H., 2008. An adaptive management approach to an octocoral fishery based on the Beverton-Holt model. Coral Reefs 27, 751-761.

Gulland, J.A., Holt, S.J., 1959. Estimation of growth parameters for data at 
unequal time intervals. Journal du Counseil International pour l'Explorations de la Mer 25, 47-49.

Kooijman, S.A.L.M., Sousa, T., Pecquerie, L., van der Meer, J., Jager, T., 2008. From food-dependent statistics to metabolic parameters. a practical guide to the use of dynamic energy budget theory. Journal of Statistical Planning and Inference 83, 533-552.

Koojiman, S.A.L.M., 2000. Dynamic energy and mass budgets in biological systems (2nd rev. ed.). Cambridge Univ. Press, Cambridge.

Lindstrom, M.J., Bates, D.M., 1990. Nonlinear mixed effects models for repeated measures data. Biometrics 46, 673-687.

Lirman, D., Formel, N., Schopmeyer, S., Ault, J.S., Smith, S.G., Gilliam, D., Riegl, B., 2014. Percent recent mortality (prm) of stony corals as an ecological indicator of coral reef condition. Ecological Indicators 44, 120-127.

Lloyd-Jones, L.R., Wang, Y., Nash, W.J., 2014. Generalised growth models for aquatic species with an application to blacklip abalone (haliotis rubra). Ecological Modelling 272, 311-322.

McClanahan, T.R., Cinner, J.E., Graham, N.A.J., Daw, T.M., Maina, J., Stead, S.M., Wamukota, A., Brown, K., Venus, V., Polunin, N., 2009. Identifying reefs of hope and hopeful actions: Contextualizing environmental, ecological, and social parameters to respond effectively to climate change. Conservation Biology 23, 662-671.

Meesters, W., Hilterman, M., Keetman, E.K.M., de Vries M, M., 2004. Colony size-frequency distributions of scleractinian coral populations: spatial and interspecific variation. Marine Ecology Progress Series 209, 43-54. 
Paul, S., Saha, K.K., 2007. The generalized linear model and extensions: a review and some biological and environmental applications. Environmetrics 18, 421-443. Doi: 10.1002/env.849.

Pignotti, E., 2013. Parameter estimation in a growth model for a biological population. Ph.D. thesis. Metodologia statistica per la ricerca scientifica. Alma Mater Studiorum Università di Bologna. http://10.6092/unibo/ amsdottorato/5169.

Pinheiro, J., Bates, D., DebRoy, S., Sarkar, D., R Core Team, 2016. nlme: Linear and nonlinear mixed effects models URL: http://CRAN.R-project. org/package=nlme, r package version 3.1-128.

Pinheiro, J.C., Bates, D.M., 2000. Mixed-Effects Models in S and S-PLUS. Springer, New York.

Purcell, S.W., Piddocke, T.P., Dalton, S.J., Wang, Y., 2016. Movement and growth of the coral reef holothuroids Bohadschia argus and Thelenota ananas. Marine Ecology Progress Series 551, 201-214.

Ricker, W., 1979. Growth rates and models. In: W.S. Hoar and D.J. Randall and J.R. Brett (Eds). Fish Physiology, vol. VIII, Bioenergetics and Growth. New York: Academic Press Inc. pp. 678-743.

Sakamoto, Y., Ishiguro, M., Kitagawa, G., 1986. Akaike information criterion statistics. Dordrecht: Reidel.

Schaalje, G.B., Shaw, J.L., Belk, M.C., 2001. Using nonlinear hierarchical models for analyzing annulus-based size-at-age data. Can. J. Fish. Aquat. Sci. 59, 1524-1532. Doi: 10.1002/env.3170060108.

Schwarz, G., 1978. Estimating the dimension of a model. Annals of Statistics $6,461-464$. 
Stolarski, J., Meibom, A., Przenioslo, R., Mazur, M., 2007. A cretaceous scleractinian coral with a calcitic skeleton. Science 318, 92-94.

von Bertalanffy, L., 1938. A quantitative theory of organic growth (inquiries on growth laws. II). Human Biology 10, 181-213.

Vonesh, R.F., Chinchilli, V.M., 1997. Linear and Nonlinear Models for the Analysis of Repeated Measurements. Marcel Dekker, Inc., New York, NY.

Yee, S.H., Barron, M.G., 2010. Predicting coral bleaching in response to environmental stressors using 8 years of global-scale data. Environmental Monitoring and Assessment 161, 423-438. 